\title{
Performance and Survivorship of National Football League Players with Pectoralis Major Injuries
}

\author{
Blake M. Bodendorfer, M.D., Steven F. DeFroda, M.D., Henry T. Shu, B.S., \\ Derrick M. Knapik, M.D., Daniel S. Yang, B.S., and Nikhil N. Verma, M.D.
}

\begin{abstract}
Purpose: The purpose of this study was to determine return-to-play (RTP), performance and career survivorship for National Football League (NFL) athletes sustaining pectoralis major (PM) injuries with comparison among grades of injury and between nonoperative and operative management. Methods: Publicly available data from the 1998-2020 NFL seasons were reviewed to identify athletes with PM injuries. Athlete characteristics were collected 1 season before and 2 seasons after injury. Percent of total games played in a season, player efficiency rating (PER), and Pro Football Focus (PFF) grades were compared for the preinjury season and 2 postinjury seasons. Kaplan-Meier survivorship plots were computed for RTP and postinjury career length, whereas a log-rank test was used to compare survivorship differences. Results: In total, 258 PM injuries were reported at a mean age of $27.1 \pm 3.3$ years. A total of 126 surgical repairs occurred in $48.8 \%$ $(\mathrm{n}=126)$ of injuries, with athletes undergoing repair possessing a lower RTP rate and longer time to RTP compared to athletes treated conservatively $(P<.001)$. Survival analysis revealed shorter career length for athletes sustaining PM tears compared to strains $(P<.001)$, although no difference in career length was appreciated on the basis of injury management $(P=.980)$. Defensive linemen and wide receivers had lower PER during their second postinjury seasons $(P=.019$ and .030 , respectively), whereas defensive linemen had lower PFF grades during their second post-injury seasons $(P=.044)$. Conclusion: NFL athletes requiring PM repair may experience a lower likelihood of RTP, and longer RTP timing, likely because of higher-grade injuries. Defensive linemen and wide receivers experiencing PM injuries are at risk for diminished performance post-injury. Career length does not appear to be affected based on injury management. Level of Evidence: Level III, cohort study.
\end{abstract}

$\mathbf{P}$ ectoralis major (PM) injuries are uncommon injuries that generally occur during weightlifting when an eccentric load is placed on the arm with the shoulder in extension, abduction, and external rotation. ${ }^{1,2}$ A subset of PM injuries has been reported during sports competition, such as American football. ${ }^{1,3-13}$ Recent evidence supports repair over nonoperative management for complete PM tears, with operatively treated patients demonstrating superior functional outcomes, strength, and cosmesis when compared to those treated conservatively. $1,2,4,14-17$

From Midwest Orthopaedics at Rush (B.M.B., S.F.D., D.M.K., N.N.V.), Chicago, IL, School of Medicine, The Johns Hopkins University (H.T.S.), Baltimore, MD, and the Warren Alpert Medical School, Brown University (D.S.Y.), Providence, RI.

The authors report the following potential conflicts of interest or sources of funding: N.N.V. reports research support from Arthrex, Inc., Breg, Ossur, Smith $\theta$ Nephew, and Wright Medical Technology, Inc.; publishing royalties, financial, or material support from Arthroscopy and Vindico MedicalOrthopedics Hyperguide; stock or stock options from Cymedica, Minivasive, and Omeros; and IP royalties from Smith $\theta$ Nephew; is a board or committee member for the American Orthopaedic Society for Sports Medicine, American Shoulder and Elbow Surgeons, Arthroscopy Association of North America; is on the editorial or governing board for Knee and SLACK Incorporated; and is
However, there remains a role for nonoperative management and physical therapy for athletes with lower grade PM strains and partial tears; however, comparative studies are lacking. 1,15,16

The PM muscle is a fan-shaped muscle over the anterior thorax with 2 distinct heads: a sternocostal head and clavicular head. 18,19 The sternocostal head arises from the sternum and ribs and the clavicular head arises from the proximal half of the clavicle. $1,18,19$ These heads converge into a tendon distal to the axilla,

a paid consultant for Minivasive and Orthospace. Full ICMJE author disclosure forms are available for this article online, as supplementary material.

Received December 18, 2020; accepted March 30, 2021.

Address correspondence to Blake M. Bodendorfer, M.D., Midwest Orthopaedics at Rush, 1611 W Harrison St., Chicago, IL 60612, U.S.A. E-mail: BlakeBodendorfer@gmail.com

(C) 2021 THE AUTHORS. Published by Elsevier Inc. on behalf of the Arthroscopy Association of North America. This is an open access article under the CC BY-NC-ND license (http://creativecommons.org/licenses/by-nc-nd/4.0/). 2666-061X/202024

https://doi.org/10.1016/j.asmr.2021.03.015 
inserting on the lateral lip of the bicipital groove. ${ }^{20-22}$ The PM has a large insertional footprint, which is typically 63 to $68 \mathrm{~mm}$ in length from proximal to distal. ${ }^{20}$ For National Football League (NFL) athletes, the PM is essential to ensure full athletic potential, functioning as a powerful adductor, internal rotator, and flexor of the humerus, providing strength and range of motion to meet the competition demands of multiple positions. ${ }^{23,24}$ Athletes with PM injuries typically present with deformity of the anterior axillary fold, ecchymosis of the arm, and weakness with resisted adduction and internal rotation accompanied by an acute and sharp sensation. ${ }^{1,19}$ Although physical examination may be sufficient to identify large PM ruptures, confirmation and assessment of the extent of PM injuries typically involve ultrasonography and magnetic resonance imaging, with the latter providing a more comprehensive assessment of the entire PM muscle and tendon. ${ }^{25}$ Current evidence favors surgical repair of some partial (grade II) and most complete (grade III) PM injuries at the tendinous insertion or musculotendinous junction or when the patient is unable to accept the resulting deformity or weakness. 2,6,26,27

In the NFL, PM injuries are infrequently reported. However, an increase in the incidence of PM injuries in the NFL has recently been reported over the past several seasons. ${ }^{27-29}$ This increase has been suggested to be secondary to the increasing size and speed of athletes during competition. ${ }^{30,31}$ Despite the increased interest in the epidemiology of PM injuries, there remains a paucity of data on return to play (RTP), performance, and survivorship after PM injuries in NFL athletes. The purpose of this study was to determine RTP, performance and career survivorship for NFL athletes sustaining PM injuries with comparison among grades of injury and between nonoperative and operative management. We hypothesized that athletes with higher-grade PM injuries and injuries undergoing repair would be associated with lower RTP, performance, and career length.

\section{Methods}

\section{Injury Data Collection}

Publicly available data from the Entertainment and Sports Programming Network ${ }^{32}$ and Pro Football Reference $^{33}$ were reviewed to capture all PM injuries reported in NFL athletes from the 1998 season to 2020 season. This method of data collection has been used in many previous publications. ${ }^{29,34-38}$ Data collection began with querying publicly available data from online league and player databases for weekly regular season NFL injury reports and identifying injuries reported as "pectoral," "chest," and "shoulder." ${ }^{32,33}$ Athlete characteristics (age, body mass index $[\mathrm{BMI}]$, years of experience, position, degree of tear, and operative versus nonoperative management) were recorded.
NFL injury reports consist of practice reports, which captures player availability during NFL practices, and game status reports, which captures player availability during games. ${ }^{39}$ All teams are mandated by the league to publish injury reports after each regular season practice and before each regular season game. ${ }^{39}$ To capture the true incidence of PM injuries, Pro-Football-Reference. com (Sports Reference LLC, Philadelphia, PA) was used to identify all "pectoral," "chest," and "shoulder" injuries that resulted in a player being designated as injured reserve or physically unable to perform. ${ }^{39}$ Once all athletes with PM injuries were identified, athletes sustaining injuries were verified by reviewing publicly available press releases. All injury dates and nonoperative versus operative treatment were identified by publicly available news articles and reports. PM injuries were graded as I (strain), II (partial tear), or III (complete tear) based on press releases and/or inferred based on return to play (RTP) and treatment. If a news report had stated that a player had a PM rupture or complete tear, it was classified as a grade III injury. If a news report had stated that a player had a partial tear, then it was classified as a grade II injury. If a news report had stated that a player had a strain or minor injury to the PM, it was classified as a grade I injury. In cases where there were no news reports, nonoperatively managed injuries with RTP within 1 season were classified as grade I injuries. Additionally, PM injuries without detailed news reports were considered grade II if they were nonoperatively managed but required the player being designated on injured reserve or as physically unable to perform. Finally, surgically managed PM injuries without detailed news reports were classified as grade III unless otherwise specified by press releases (e.g., reports of players being treated with repair for grade II PM injury).

\section{Player Efficiency Rating Data Collection}

The modified player efficiency rating (PER) for football uses games played, games started, seasons played, sacks, interceptions, yards gained, and touchdowns to generate a single score for player rating. These statistics were collected from Pro-Football-Reference.com (Sports Reference LLC), a publicly available database with player and team statistics. The algorithm used to calculate the PER from collected statistics has been previously reported by Hsu. ${ }^{34}$ PER statistics were collected and scores were calculated 1 season before PM injury and the subsequent 2 postinjury seasons using only regular season data. The first postinjury season was defined as the immediate season that the player was able to RTP after injury.

\section{Pro Football Focus Data Collection}

Given the limitations in PER in generating quantitative scores in offensive linemen, Pro Football Focus (PFF) (PFF, Cincinnati, OH) was used to collect 
Table 1. Demographics by Player Position and Treatment

\begin{tabular}{|c|c|}
\hline Overall & Mean \pm SD [Range] \\
\hline$\overline{\text { Age }}$ & $27.1 \pm 3.3$ years $[21.0-37.0]$ \\
\hline BMI & $32.6 \pm 4.1[24.8-43.1]$ \\
\hline Nonoperatively Treated & $116(45.0 \%$ of 258$)$ \\
\hline Age & $27.4 \pm 3.3$ years $[21.0-37.0]$ \\
\hline BMI & $32.7 \pm 4.0[24.9-41.9]$ \\
\hline Defense & $60(51.2 \%$ of 116$)$ \\
\hline Defensive line & 21 \\
\hline Linebacker & 24 \\
\hline Defensive back/secondary & 15 \\
\hline Offense & $56(48.3 \%$ of 116$)$ \\
\hline Offensive line & 30 \\
\hline Running back & 7 \\
\hline Wide receiver & 6 \\
\hline Tight end & 8 \\
\hline Quarterback & 5 \\
\hline Special Teams & $0(0.0 \%$ of 116$)$ \\
\hline Operatively Treated & $126(48.8 \%$ of 258$)$ \\
\hline Age & $26.8 \pm 3.3$ years $[22.0-37.0]$ \\
\hline BMI & $32.5 \pm 4.0[25.3-43.1]$ \\
\hline Defense & $98(77.8 \%$ of 126$)$ \\
\hline Defensive line & 26 \\
\hline Linebacker & 46 \\
\hline Defensive back/secondary & 26 \\
\hline Offense & $27(21.4 \%$ of 126$)$ \\
\hline Offensive line & 18 \\
\hline Running back & 5 \\
\hline Wide receiver & 0 \\
\hline Tight end & 1 \\
\hline Quarterback & 3 \\
\hline Special Teams & $1(0.8 \%$ of 126$)$ \\
\hline
\end{tabular}

Player demographics in the overall group and between operatively treated and nonoperatively treated players. There were a total of 258 injuries; however, 16 players had unknown treatments, leaving a total of 242 players with known operative or nonoperative treatment. BMI, body mass index; SD, standard deviation,

additional quantitative player grades for analysis. ${ }^{40} \mathrm{PFF}$ provides an overall offensive or defensive performance score for each player for each season based on performance assessed by video analysis. ${ }^{40}$ Every play a player makes is manually reviewed by a team of analysts, who grades each play attempt based on a scale of -2 to +2 in 0.5 increments. The scores are then converted to a scale of 0 to 100, with 0 being the worst score and 100 being the best score. The overall offensive or defensive season rating on the 0 to 100 scale was recorded. PFF player grades were again collected 1 season before PM injury and 2 seasons after injury.

\section{Data Analysis and Statistical Methods}

Percent of total games played in a season, PER, and PFF were calculated for the preinjury season and postinjury seasons 1 and 2 and expressed as mean and 95\% confidence interval (CI). To identify statistical significance between RTP rates based operative versus nonoperative treatment, an unpaired Student $t$-test with unequal variance was used. To identify statistical significance between RTP rates in operatively treated versus nonoperatively treated players, a Student $t$-test for proportions was used. For both PFF and PER, the percent change from the preinjury to each postinjury period was calculated and compared with paired Student's $t$-tests with equal variance. Kaplan-Meier survivorship plots were computed for RTP and postinjury career length. Log-rank test compared differences between survivorship across stratified cohorts. Statistical analysis was performed using RStudio, Version 1.1.442 (RStudio Inc. Boston, MA). Values are demonstrated as mean (range), mean \pm standard deviation (SD), or mean (confidence interval [CI]) when appropriate. An $\alpha$ value of .05 was used.

\section{Results}

In total, 258 PM injuries in 245 players were identified between the 1998-1999 and 2019-2020 NFL seasons. Mean age in athletes at the time of injury was $27.1 \pm 3.3$ years, whereas mean BMI was $32.6 \pm 4.1$. Player demographics are demonstrated in Table 1. The majority of recorded PM injuries were in defensive players (167 of $258,64.7 \%$ ), with linebacker being the most commonly injured position (73 of 167 defensive injuries, $43.7 \%$ ). After injury, $48.8 \%$ (126 of 258 ) of injuries underwent repair, with complete tears representing $95.2 \%$ of the operative group (120 of 126). Additionally, $45.0 \%$ of injuries (116 of 258) were treated without surgery, and $6.2 \%$ (16 of 258) were indeterminately treated. For those that were operatively treated with known surgery dates, the mean \pm SD time from injury to surgery was $6.8 \pm 9.6$ days (range, 2.0-59.0 days). No significant difference in athlete age $(P=.194)$ and BMI $(P=.779)$ were appreciated when comparing athletes undergoing nonoperative versus operative management. The majority of operatively treated players were defensive players $(77.8 \%$ of 126$)$, with linebacker being the most common defensive position undergoing repair. The most common operatively treated offensive position was the offensive lineman. A slightly larger proportion of defensive players $(51.2 \%)$ were nonoperatively treated as compared to offensive players $(48.3 \%)$.

Of the $48.8 \%$ players (126 of 258 ) that were operatively treated, $95.2 \%$ (120 of 126) had grade III injuries. $4.2 \%$ of operatively treated injuries (5 of 126 ) were grade II tears, while none were grade I. Of the $45.0 \%$ of players nonoperatively treated (116 of 258), no athlete possessed a grade III injury. Of the nonoperatively treated injuries, $76.7 \%$ (89 of 116) were grade I tears and $23.3 \%$ (27 of 116 ) were grade II tears (Table 2 ).

Overall, $85.3 \%$ players (220 of 258 ) returned to competition following PM injury, with a mean time to RTP of $165.8 \pm 147.9$ days (range 2-668 days). Athletes undergoing repair had a RTP rate of $78.6 \%$ (CI 70.4$85.4 \%$ ) versus $95.7 \%$ (CI $90.2-98.6 \%$ ) for nonoperatively treated players $(P<.005)$. One player with 
Table 2. Pectoralis Major Tear Degree by Treatment Received

\begin{tabular}{lccccc}
\hline & Total Players & Grade I Tear & Grade II tear & Grade III Tear & Unknown Tear Degree \\
\hline Operative treatment & $126(48.8 \%)$ & 0 & $5(4.2 \%)$ & $120(95.2 \%)$ & $1(0.8 \%)$ \\
Nonoperative treatment & $116(45.0 \%)$ & $89(76.7 \%)$ & $27(23.3 \%)$ & 0 & 0 \\
\hline
\end{tabular}

The degree of tears that operatively and nonoperatively treated players sustained.

a complete tear had RTP of 14 days due to desire to play through the rest of his season with the injury before undergoing elective repair at the conclusion of the season. RTP timing was also significantly different in operatively treated players returning at 289.5 (CI 269.5-309.5) days post-injury versus nonoperatively treated players returning at 47.4 (CI 33.9-60.9) days post-injury $(P<.001)$. Athletes with higher injury grades (grades II and III) had reduced RTP rates, with athletes with grade I injuries possessing a RTP rate of $97.8 \%$, grade II injuries having a RTP of $87.9 \%$, and grade III injuries having a RTP of $78.0 \%$. Additionally, higher grades of injury were associated with longer RTP, with grade I injuries having mean RTP timing of $30.4 \pm 42.5$ days (range, $2-259$ days), $117.4 \pm 111.5$ days (range, 6-420 days) for grade II injuries, and 297.5 \pm 89.1 days (range, 69-668 days) for grade III injuries. Cumulative RTP analysis demonstrated significantly longer RTP time for athletes undergoing surgical repair versus nonoperatively treated athletes $(P<.001$, Fig 1$)$ and for athletes with grade II and grade III injuries compared to grade I injuries $(P<.001$ for both, Fig 2$)$.

Regarding playing time, when all players were considered as a group, athletes played in fewer games both in their first and second post-injury seasons compared to pre-injury season (Table 3). However, when analyzed alone, operatively treated players did not play in significantly fewer games during either their first or second postinjury seasons compared to preinjury seasons. Additionally, athletes undergoing nonoperative management and athletes with grade II injuries played in significantly fewer games in the first postinjury season; however, this difference was not present during the second postinjury season. Overall, defensive athletes played in significantly fewer games in both their first and second seasons post-injury, whereas linebackers and quarterbacks only played in significantly fewer games in their first post-injury seasons (Table 4). Additionally, defensive linemen played in significantly fewer games in their second, but not first, postinjury seasons. Although nonoperatively treated players played in significantly fewer games in the first postinjury season $1(P=.043)$, they returned to preinjury levels at the second postinjury season $(P=.795)$. Operatively treated players averaged 2.22 (CI 1.84-2.60) postinjury seasons whereas nonoperatively treated players averaged 2.65 (CI 2.18-3.12) seasons. Survival analysis showed no difference in retirement survival between operative and nonoperative players $(P=.980)$, as well as for degree of injury $(P=.540)$.

For both operatively and nonoperatively treated players, PER was not significantly different at the first and second postinjury seasons. Additionally, for each grade of injury, PER was not significantly different at the first and second postinjury seasons. However, when all injuries were combined, there was a significantly

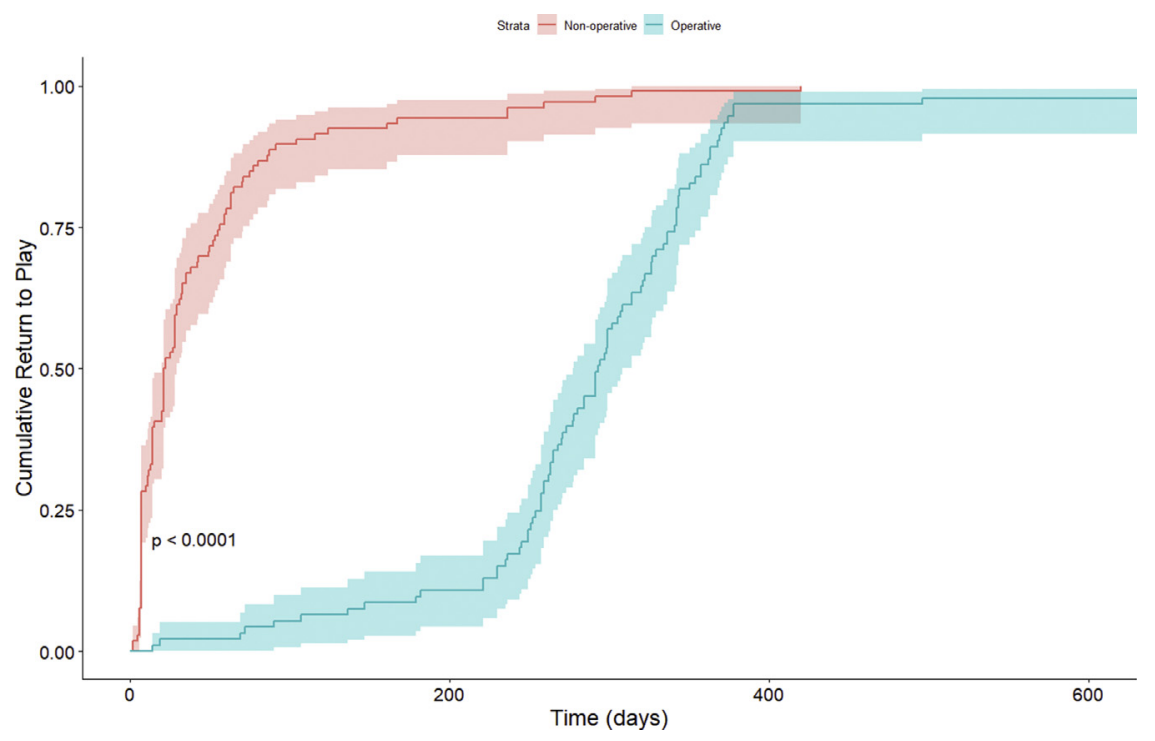

Fig 1. The Kaplan-Meier survivorship analysis of return-to-play of operatively treated versus nonoperatively treated players with pectoralis major injuries. $P$ value is from a logrank test between both groups. 
Fig 2. The Kaplan-Meier survivorship analysis of return-to-play of players with grade 1 (strains), grade 2 (partial tear), and grade 3 (full tear) pectoralis major injuries. $P$ value is from log-rank test between each group.

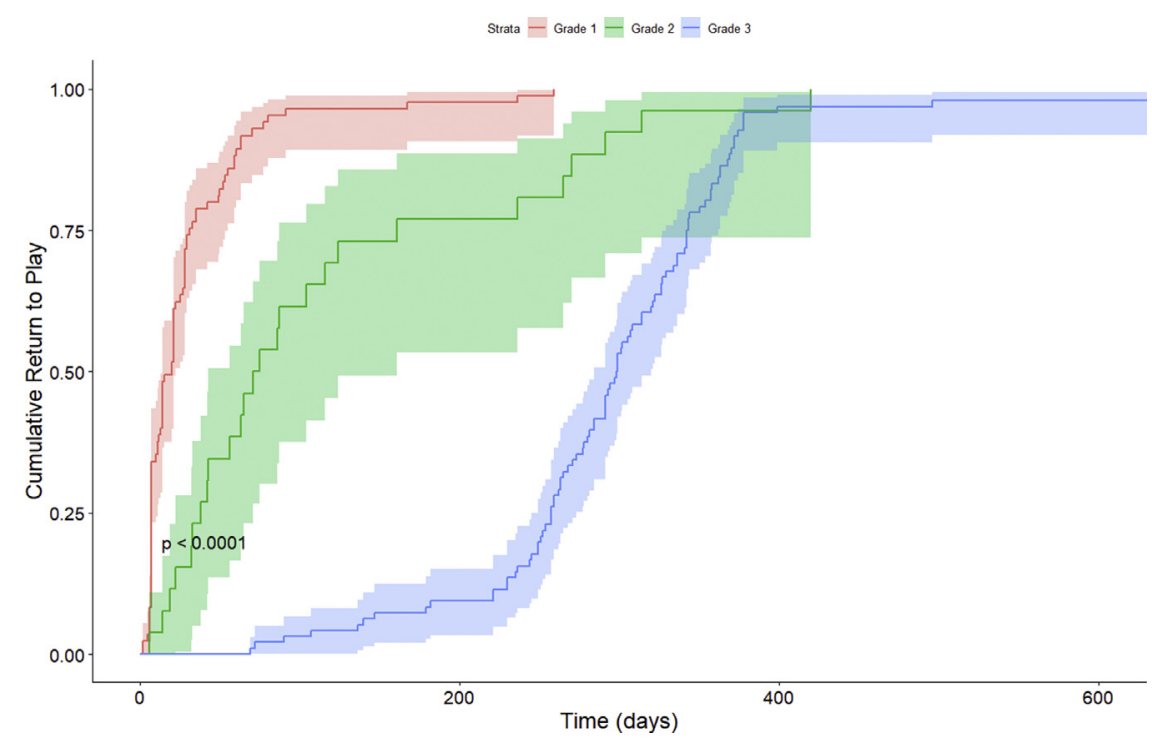

improved PER when comparing PER in the first and second postinjury seasons (1.07, CI .62-1.51 vs 1.19, CI $.60-1.70)(P=.022)$, indicating that athletes generally improved in performance in the subsequent season after returning to play when compared to the first postinjury season. Preinjury PER of athletes sustaining injuries that required operative treatment was significantly lower than that of athletes who had nonoperative injuries $(P=.004)$. PER scores were significantly lower for overall defensive players and defensive backs in the first post-injury seasons (Table 5). PER scores were significantly lower for defensive linemen and wide receivers in the second post-injury seasons (Table 5). Defensive linemen also had significantly lower PFF player grades in the second postinjury seasons (66.6 points, CI 63.4-69.8 vs 62.8 points, CI 59.2-66.4) $(P=.044)$. However, none of the other positions had significantly different PFF player grades after injury.

\section{Discussion}

Regarding PM tears in NFL players, operatively treated players had both a significantly lower and longer RTP time when compared to athletes undergoing conservative management. However, this may be due to the severity of injury, rather than the treatment itself, because the majority of operatively treated injuries were grade III injuries. It is important to note that PM injury grading was primarily determined by news reports, and, if news was unavailable, then it was estimated on the basis of treatment. Athletes with a higher injury grade also possessed a longer time to RTP. Concerning player performance, PER scores were not significantly different 1 or 2 seasons postinjury after operative or nonoperative treatment, suggesting that treatment may equalize player performance potential. This may be further supported by the finding that operatively treated players did not play in significantly fewer games in their first or second postinjury seasons. Similarly, career survivorship was not dependent on injury grade or injury management.

This study demonstrates that only defensive linemen and wide receivers had significant performance reductions within the first 2 postinjury seasons. The differential effect of PM injury on performance based on

Table 3. Percentage of Games Played by Treatment and Tear Degree*

\begin{tabular}{|c|c|c|c|c|c|}
\hline & Preinjury Season & Postinjury Season 1 & $P$ Value $^{\dagger}$ & Postinjury Season 2 & $P$ Value ${ }^{\dagger}$ \\
\hline All injuries & $81.7 \%(78.4-85.0)$ & $77.9 \%(73.5-82.3)$ & .017 & $79.4 \%(75.0-83.8)$ & .022 \\
\hline Nonoperative & $84.7 \%(80.4-89.1)$ & $78.8 \%(72.3-85.2)$ & .043 & $82.6 \% \quad(76.8-88.4)$ & .392 \\
\hline Operative & $79.5 \%(74.4-84.6)$ & $78.4 \%(72.3-84.5)$ & .280 & $76.6 \% \quad(69.6-83.6)$ & .059 \\
\hline Grade 1 tear & $81.7 \% \quad(76.6-86.8)$ & $79.4 \%(72.0-86.8)$ & .350 & $81.1 \%(74.1-88.1)$ & .360 \\
\hline Grade 2 tear & $88.5 \% \quad(79.4-97.6)$ & $78.5 \%(66.4-90.6)$ & .041 & $80.5 \%(68.1-92.9)$ & .372 \\
\hline Grade 3 tear & $79.8 \% \quad(74.9-84.7)$ & $77.3 \%(71.0-83.6)$ & .176 & $78.2 \%(71.4-85.0)$ & .080 \\
\hline
\end{tabular}

The percentage of games played by players before injury, l season after injury, and 2 seasons after injury stratified by operative versus nonoperative treatment and degree of tear. Bold indicates $P<.05$.

$* 95 \%$ confidence intervals are expressed parenthetically following means.

${ }^{\dagger} P$-values are from 2 -sample paired $t$-tests to the preinjury season. 
Table 4. Percentage of Games Played by Position*

\begin{tabular}{|c|c|c|c|c|c|}
\hline & Pre-Injury Season & Post-Injury Season 1 & $P$-value ${ }^{\dagger}$ & Post-Injury Season 2 & $P$-value ${ }^{\dagger}$ \\
\hline Overall defense & $83.4 \%(79.6-87.2)$ & $79.0 \%(73.9-84.1)$ & .018 & $81.2 \%(76.1-86.3)$ & .032 \\
\hline Defensive line & $86.3 \%(79.7-92.9)$ & $79.7 \%(71.4-88.0)$ & .097 & $76.9 \%(68.6-85.2)$ & .005 \\
\hline Defensive back & $77.8 \%(68.7-86.9)$ & $79.5 \%(67.6-91.4)$ & .920 & $82.9 \%(70.2-95.6)$ & .747 \\
\hline Overall offense & $78.1 \%(71.8-84.4)$ & $75.4 \%(67.0-83.8)$ & .430 & $76.3 \%(67.8-84.8)$ & .378 \\
\hline Offensive line & $83.4 \%(76.0-90.8)$ & $76.6 \%(65.7-87.5)$ & .230 & $83.9 \%(73.4-94.4)$ & .419 \\
\hline Quarterback & $45.5 \%(12.9-78.1)$ & $43.8 \%(-5.8-93.4)$ & .041 & $34.4 \%(-15.2-84.0)$ & .372 \\
\hline Wide receiver & $78.6 \%(55.0-102.2)$ & $95.0 \%(84.9-105.1)$ & .405 & $85.0 \%(54.6-115.4)$ & .742 \\
\hline
\end{tabular}

The percentage of games played by players pre-injury, l season post-injury, and 2 seasons post-injury stratified by position. Bold indicates $P<.05$.

$* 95 \%$ confidence intervals are expressed parenthetically following means. There were too few players for special teams analysis.

${ }^{\dagger} P$ values are from 2 -sample paired $t$-tests to the preinjury season.

position may be related to the varying demands that each position requires of the upper extremity. For defensive lineman, blocking and tackling are essential skills. Tackling and blocking requires forceful shoulder flexion in an extended, abducted, and externally rotated position, which eccentrically loads the PM. ${ }^{41,42}$ Thus persistent weakness in the PM muscle may limit the blocking or tackling potential of an NFL athlete. Alternately, for wide receivers, PM activation may be necessary in high-speed catching or in stabilizing the shoulder girdle during a stiff-arm fend. ${ }^{43}$

The present study demonstrated an overall RTP after PM injury to be $85.3 \%$. However, operatively treated players had significantly reduced RTP rate when compared with nonoperatively treated players. As previously discussed, this difference is likely due to the greater magnitude of injury sustained by players requiring operative treatment (Table 2 ). In a systematic review, Yu et $\mathrm{al}^{44}$ reported that $90 \%$ of patients undergoing PM repair successfully returned to sport, with most of those patients being weightlifters. However, only $8 \%$ of these were professional athletes. ${ }^{44}$ The relatively lower RTP of $78.6 \%$ in operatively treated NFL athletes in this study is likely due to the high physical demands of professional American football. Similarly, Wise and Gallo ${ }^{29}$ reported an $85.4 \%$ RTP in 55 NFL players with PM ruptures. ${ }^{29}$ Recently, Sahota et al. ${ }^{27}$ examined the incidence of PM injuries, including strains, partial tears, and complete ruptures, from the 2000-2014 NFL seasons. Similar to the present study, Sahota et al. ${ }^{27}$ identified that operatively treated PM injuries resulted in significantly longer RTP times as compared to nonoperatively treated injuries. Sahota et al. $^{27}$ identified mean days missed because of PM strains to be 28.3 days, and ruptures to be 129.6 days. This was less than the time to RTP reported for both types of injuries in this study. ${ }^{27}$ This difference may be due to the strict definition of RTP used in this study, which is the point at which the player returned to full competition, versus days missed due to injury used in Sahota et al. ${ }^{27}$ Additionally, Sahota et al. ${ }^{27}$ classified both grade I and grade II injuries as strains and only examined injuries between 2000 to 2014. Nonetheless, the difference between RTP in operatively versus

Table 5. Player Efficiency Rating (PER) by Player Position*

\begin{tabular}{|c|c|c|c|c|c|}
\hline & Pre-Injury Season & Post-Injury Season 1 & $P$ Value $^{\dagger}$ & Post-Injury Season 2 & $P$ Value $^{\dagger}$ \\
\hline Overall defense & $.32(.26-.37)$ & $.28(.23-.34)$ & .046 & $.29(.21-.37)$ & .068 \\
\hline Defensive line & $.38(.29-.47)$ & $.335(.22-.45)$ & .451 & $.27(.18-.37)$ & .019 \\
\hline Defensive back & $.35(.22-.49)$ & $.25(.13-.38)$ & .049 & $.34(.09-.58)$ & .245 \\
\hline Linebacker & $.250(.18-.32)$ & $.263(.19-.33)$ & .535 & $.284(.17-.40)$ & .916 \\
\hline Overall offense & $4.74(2.81-6.67)$ & $2.61(.41-4.80)$ & .653 & $2.79(1.37-4.21)$ & .401 \\
\hline Offensive line $e^{\ddagger}$ & - & - & & - & \\
\hline Quarterback & $10.36(2.92-17.80)$ & $11.05(-1.19-23.30)$ & .825 & $7.53(-.14-15.20)$ & .081 \\
\hline Running back & $4.45(.41-8.48)$ & $6.58(1.46-11.69)$ & .467 & $7.56(1.80-13.30)$ & .562 \\
\hline Tight end & $1.99(-1.01-5.00)$ & $2.72(-.32-5.76)$ & .770 & $2.99(-1.41-7.40)$ & .778 \\
\hline Wide receiver & $6.68(3.20-10.20)$ & $5.24(.63-9.84)$ & .291 & $3.58(-.63-7.79)$ & .030 \\
\hline
\end{tabular}

The Player Efficiency Rating of players before injury, l season after injury, and 2 seasons after injury stratified by player position. Bold indicates $P<.05$.

$* 95 \%$ confidence intervals are expressed parenthetically following means. There were too few players for special teams analysis.

${ }^{\dagger} P$-values are from 2 -sample paired t-test to the pre-injury season.

${ }^{\ddagger}$ PER Score not applicable. 
nonoperatively treated players in both the present study and the work of Sahota et al. ${ }^{27}$ demonstrates that higher grade injuries are more likely to require operative treatment and thus result in a longer RTP. Moreover, our data highlight that grade III injuries are associated with operative treatment, as no nonoperatively treated players had grade III injuries, whereas $95.2 \%$ of operatively treated players had grade III injuries.

We found that most players returned to baseline function by 1 or 2 seasons after injury with the exception of defensive linemen and wide receivers. Previous studies have examined functional outcome, with respect to percent of pre-injury bench-press, in the general population. 2,14,26,45-47 Others have also reported excellent outcomes after PM injuries undergoing operative treatment in active-duty United States military personnel and military academy students. ${ }^{48-51}$ Recently, Liu et al. ${ }^{26}$ demonstrated significantly reduced PM strength after PM injury at a mean 2-year follow-up, which is consistent with previous studies. ${ }^{2,45,52}$ A prior study demonstrated that up to $40 \%$ of surgically treated recreational athletes had a greater than $20 \%$ deficiency of isokinetic strength when compared to the uninjured contralateral side. ${ }^{15}$ This persistent loss of strength following operative treatment may explain the performance decrease following injury in some NFL players, especially for defensive players who need to tackle. Despite this, we demonstrate that repair of higher-grade injuries may be career preserving, because there was no difference between operatively and nonoperatively managed players. Thus, although there may be a persistent loss of strength after repair, this difference likely does not significantly impact the length of players' careers.

\section{Limitations}

This study is not without limitations. An inherent limitation of this study is the use of public databases to capture PM injuries. There were several NFL injuries classified as "undisclosed" or "chest" injuries that were unable to be confirmed as PM injuries. Nonetheless, the use of multiple public databases with weekly injury reports and press releases in this study allowed for us to capture all reported PM injuries, and this method has been widely reported in the literature. ${ }^{29,34-36,38,53}$ Furthermore, degree of PM injury was estimated on the basis of the news reports and, if unavailable, treatment versus physical examination and magnetic resonance imaging as has been reported using the NFL Injury Surveillance System. This makes our grading system inherently more biased than medical imaging, which we did not have access to. Nevertheless, we believe that our grading technique is still valid as many press releases specified the degree of PM injury that the player had. Also, data on surgical techniques, instrumentations and the incidence of perioperative complications were not publicly available.

\section{Conclusions}

NFL athletes requiring PM repair may experience a lower likelihood of RTP, and longer RTP timing, likely because of higher-grade injuries. Defensive linemen and wide receivers experiencing PM injuries are at risk for diminished performance after injury. Career length does not appear to be affected on the basis of injury management.

\section{References}

1. Provencher M, Handfield K, Boniquit N, Reiff S, Sekiya J, Romeo A. Injuries to the pectoralis major muscle: diagnosis and management. Am J Sports Med 2010;38:1693-1705.

2. Bodendorfer B, McCormick B, Wang D, et al. Treatment of pectoralis major tendon tears: A systematic review and meta-analysis of operative and nonoperative treatment. Orthop J Sports Med 2020;8:23259671 19900813.

3. Golshani K, Cinque M, O’Halloran P, Softness K, Keeling L, Macdonell J. Upper extremity weightlifting injuries: Diagnosis and management. J Orthop 2018;15:24-27.

4. Haley C, Zacchilli M. Pectoralis Major Injuries: Evaluation and Treatment. Clin Sports Med 2014;33:739-756.

5. Äärimaa V, Rantanen J, Heikkilä J, Helttula I, Orava S. Rupture of the pectoralis major muscle. Am J Sports Med 2004;32:1256-1262.

6. Bak K, Cameron E, Henderson I. Rupture of the pectoralis major: A meta-analysis of 112 cases. Knee Surg Sports Traumatol Arthrosc 2000;8:113-119.

7. Butt U, Mehta S, Funk L, Monga P. Pectoralis major ruptures: A review of current management. J Shoulder Elbow Surg 2015;24:655-662.

8. Cordasco F, Mahony G, Tsouris N, Degen R. Pectoralis major tendon tears: Functional outcomes and return to sport in a consecutive series of 40 athletes. J Shoulder Elbow Surg 2017;26:458-463.

9. de Castro Pochini A, Andreoli C, Belangero P, et al. Clinical considerations for the surgical treatment of pectoralis major muscle ruptures based on 60 cases: A prospective study and literature review. Am J Sports Med 2014;42:95-102.

10. Garrigues G, Kraeutler M, Gillespie R, O'Brien D, Lazarus M. Repair of pectoralis major ruptures: Singlesurgeon case series. Orthopedics 2012;35:el184-el190.

11. Hasegawa K, Schofer J. Rupture of the pectoralis major: A case report and review. J Emerg Med 2010;38:196-200.

12. Wolfe S, Wickiewicz T, Cavanaugh J, Shirley P. Ruptures of the pectoralis major muscle. An anatomic and clinical analysis. Am J Sports Med 1992;20:587-593.

13. Dick R, Ferrara MS, Agel J, et al. Descriptive epidemiology of collegiate men's football injuries: National Collegiate Athletic Association injury surveillance system, 19881989 through 2003-2004. J Athl Train 2007;42:221-233.

14. Bodendorfer B, Wang D, McCormick B, et al. Treatment of pectoralis major tendon tears: A systematic review and meta-analysis of repair timing and fixation methods. Am J Sports Med 2020;48:3376-3385.

15. Fleury A, da Silva A, Pochini A, Ejnisman B, de Lira C, Andrade M. Isokinetic muscle assessment after treatment of pectoralis major muscle rupture using surgical or nonsurgical procedures. Clinics 2011;66:313-320. 
16. Hanna C, Glenny A, Stanley S, Caughey M. Pectoralis major tears: Comparison of surgical and conservative treatment. Br J Sports Med 2001;35:202-206.

17. Kakwani R, Matthews J, Kumar K, Pimpalnerkar A, Mohtadi N. Rupture of the pectoralis major muscle: Surgical treatment in athletes. Int Orthop 2007;31:159-163.

18. Fung L, Wong B, Ravichandiran K, Agur A, Rindlisbacher T, Elmaraghy A. Three-dimensional study of pectoralis major muscle and tendon architecture. Clin Anat 2009;22:500-508.

19. Petilon J, Carr DR, Sekiya JK, Unger DV. Pectoralis major muscle injuries: Evaluation and management. J Am Acad Orthop Surg 2005;13:59-68.

20. Bois A, Lo I. Surgical anatomy of the pectoralis major tendon insertion revisited: Relationship to nearby structures and the pectoral eminence for defining the anatomic footprint. JSES Int 2020;4:324-332.

21. Jagiasi J, Valavi A, Ubale T, Sahu D. Insertion anatomy of the pectoralis major tendon. J Clin Orthop Trauma 2019;10:541-543.

22. Carey P, Owens B. Insertional footprint anatomy of the pectoralis major tendon. Orthopedics 2010;33:23.

23. Quinlan J, Molloy M, Hurson B. Pectoralis major tendon ruptures: When to operate. Br J Sports Med 2002;36:226-228.

24. Rockwood C, Wirth M, Fehringer E. Rockwood and Matsen's The Shoulder, 5th Edition. 5th ed. Philadelphia: Elsevier, 2016.

25. Lee YK, Skalski MR, White EA, et al. US and MR imaging of pectoralis major injuries. Radiographics 2017;37:176-189.

26. Liu J, Gowd A, Garcia G, et al. Analysis of return to sport and weight training after repair of the pectoralis major tendon. Am J Sports Med 2019;47:2151-2157.

27. Sahota S, Gibbs D, Lawton C, et al. Pectoralis major injuries in the National Football League. Sports Health 2020;12:116-123.

28. Tarity T, Garrigues G, Ciccotti M, et al. Pectoralis major ruptures in professional American football players. Phys Sportsmed 2014;42:131-135.

29. Wise P, Gallo R. Increasing incidence of pectoralis major ruptures in NFL players. Orthop J Sports Med 2019;7: 2325967119S00396. (7 suppl 5).

30. Adickes MS, Stuart MJ. Youth football injuries. Sports Med 2004;34:201-207.

31. DeLee JC, Farney WC. Incidence of injury in Texas high school football. Am J Sports Med 1992;20:575-580.

32. ESPN NFL injuries [Internet]. Available at: https://Www. espn.com/nfl/injuries 2020. Accessed December 26, 2020.

33. Pro-Football-Reference.com [Internet]. Available at: https://www.pro-football-reference.com/ 2020. Accessed December 26, 2020.

34. Hsu W. Performance-based outcomes following lumbar discectomy in professional athletes in the national football league. Spine 2010;35:1247-1251.

35. Hsu W. Outcomes following nonoperative and operative treatment for cervical disc herniations in national football league athletes. Spine 201 1;36:800-805.

36. Nguyen M, Hsu W. Performance-based outcomes following patellar tendon repair in professional athletes. Phys Sportsmed 2020;48:110-115.
37. Mai H, Chun D, Schneider A, et al. Performance-based outcomes after anterior cruciate ligament reconstruction in professional athletes differ between sports. Am J Sports Med 2017;45:2226-2232.

38. Minhas S, Kester B, Larkin K, Hsu W. The effect of an orthopaedic surgical procedure in the National Basketball Association. Am J Sports Med 2016;44:1056-1061.

39. The National Football League. 2017 Personnel (Injury) Report Policy 2017.

40. PFF Player Grades [Internet]. Available from: https:// premium.pff.com/nfl/ 2020. Accessed December 26, 2020.

41. Journell T. Biomechanics of TacklingHung GK, Pallis JM, eds. Biomedical Engineering Principles in Sports. Bioengineering, Mechanics, and Materials: Principles and Applications in Sports, Vol. 1 Berlin: Springer, 2004;321-331;Vol. 1, 2004.

42. Horsley I, Herrington L. Electromyographic analysis of the tackle within rugby football. Phys Ther Sport 2006;7:176.

43. D'Andola M, Cesqui B, Portone A, Fernandez L, Lacquaniti F, d'Avella A. Spatiotemporal characteristics of muscle patterns for ball catching. Front Comput Neurosci 2013;7:107.

44. Yu J, Zhang C, Horner N, et al. Outcomes and return to sport after pectoralis major tendon repair: A systematic review. Sports Health 2019;11:134-141.

45. He Z, Ao Y, Wang J, Hu Y, Yin Y. Twelve cases of the pectoralis major muscle tendon rupture with surgical treatment-An average of 6.7-year follow-up. Chin Med J 2010;123:57-60.

46. Mooers B, Westermann R, Wolf B. Outcomes following suture-anchor repair of pectoralis major tears: A case series and review of the literature. Iowa Orthop J 2015;35: 8-12.

47. Schepsis A, Grafe M, Jones H, Lemos M. Rupture of the pectoralis major muscle: Outcome after repair of acute and chronic injuries. Am J Sports Med 2000;28:9-15.

48. Antosh I, Grassbaugh J, Parada S, Arrington E. Pectoralis major tendon repairs in the active-duty population. Am J Orthop 2009;38:26-30.

49. Chan A, Balazs G, Haley C, Posner M, Rue J, Owens B. Pectoralis major rupture in military academy athletes. Orthop J Sports Med 2019;7:2325967119860157.

50. Salazar D, Davis W, Shakir I, Joe K, Choate W. Acute pectoralis major tears in active duty US military personnel: Midterm outcomes of repairs performed in the forward-deployed setting. J Orthop Surg 2019;27.

51. Shakir I, Davis W, Choate W, Antosh I, Parada S, Salazar D. Outcomes of pectoralis major tears in active duty US military personnel: A comparison of surgical repairs performed in the forward deployed setting to those performed in the continental United States. Mil Med 2019;184:e802-e807.

52. Guity M, Vaziri A, Shafiei H, Farhoud A. Surgical treatment of pectoralis major tendon rupture (outcome assessment). Asian J Sports Med 2014;5:129-135.

53. Hsu W, McCarthy K, Savage J, Roberts D, Roc G, Micev A, et al. The Professional Athlete Spine Initiative: Outcomes after lumbar disc herniation in 342 elite professional athletes. Spine J 2011;11:180-186. 